\title{
La formation des maîtres de géographie aux «Etudes pédagogiques de l'enseignement secondaire» à Genève
}

\section{Conditions générales de la formation}

A Genève, ce sont les «Etudes pédagogiques de l'enseignement secondaire» (EPS) qui assurent la formation initiale des maîtres qui se destinent (ou qui enseignent déjà) aux degrés 7 à 9 et/ou 10 à 13 de l'enseignement. Bien qu'il soit possible d'enseigner la géographie dès que l'on possède une demi-licence universitaire dans cette discipline, à titre de "suppléant» (principe d'un engagement annuel, sans garantie d'emploi), il faut être porteur d'une licence universitaire en géographie pour accéder à la formation pédagogique qui mène à l'obtention d'un CAES (Certificat d'aptitude à l'enseignement secondaire).

La durée de la formation pédagogique est en principe de deux ans. C'est une formation en emploi, c'est-à-dire que le candidat assure un enseignement d'une dizaine d'heures par semaine. Sous certaines conditions, les maîtres qui disposent déjà d'une longue expérience pratique peuvent suivre une formation aménagée, d'une seule année. Actuellement, la nomination dans l'enseignement secondaire genevois intervient en principe dans les trois années qui suivent la fin de la formation pédagogique. La formation aux EPS, pour les candidats géographes comme pour la plupart des autres candidats, comprend les modules suivants:

\section{$\rightarrow$ en lre année:}

52 heures de méthodologie de la discipline (appelée «didactique de la discipline» dans d'autres cantons romands);

52 heures de didactique générale et de pédagogie;

24 heures de psychologie;

12 heures de sociologie.

Au total, le candidat participe à quelque 140 heures de séminaires dits intégrés, dans la mesure où les formateurs sont appelés à collaborer dans une perspective inter- et transdisciplinaire. Ces séminaires sont regroupés sur une même demi-journée, selon une alternance définie par l'ensemble de l'équipe des formateurs.

Parmi les formateurs, seuls le(s) méthodologue(s) observe(nt) régulièrement les candidats dans leurs classes. A la suite de chaque visite, un rapport est rédigé, discuté lors d'un entretien personnel, puis remis au candidat. A la fin du premier semestre et en fin d'année, des rapportsbilan sont établis.
Le candidat doit également suivre deux stages actifs dans des types d'école et des degrés d'enseignement différents, en tout entre 70 et 80 heures. Environ un cinquième des heures de stage sont données par le candidat. Les maîtres de stage ont un rôle formatif important; à la fin du stage, ils remettent un rapport d'évaluation.

Le parcours de formation étant assez souple, le candidat de 1 re année peut anticiper certaines des exigences de $2 \mathrm{e}$ année.

\section{$\rightarrow$ en 2e année:}

Le candidat conserve une suppléance de 10 à 12 heures par semaine et participe à deux autres stages actifs (60 heures). Il continue à recevoir les visites du méthodologue.

Sur le plan des séminaires:

- il suit 12 heures de méthodologie de la discipline;

- il participe à quatre demi-journées «d'informations générales», qui correspondent à une sensibilisation dans des domaines à choix (toxicodépendance des adolescents, accueil des immigrés et éducation interculturelle, relation famille - école, utilisation de l'ordinateur, ...);

- il suit une formation aux moyens audiovisuels dans le cadre d'ateliers dédiés (réalisation d'acétates pour le rétroprojecteur ou de diapositives, techniques infographiques ou vidéo, par exemple). Il réalise un travail personnel relié concrètement à son enseignement, en utilisant une ou plusieurs des techniques étudiées;

- il choisit deux séminaires à option semestriels ou un séminaire annuel, parmi un vaste choix qui comprend certains cours universitaires de la Faculté de Psychologie et des Sciences de l'Education de l'Université de Genève.

Pierre Speck, 6, ch. Commanderie, 1228 Plan-les-Ouates 
2. La méthodologie:

des aller et retour entre théorie et pratique

Le séminaire de méthodologie de géographie poursuit plusieurs buts simultanément:

- Il doit répondre, d'une part, aux besoins immédiats des candidats, dans leur pratique scolaire quotidienne.

- Il doit refléter, d'autre part, la diversité des pratiques dans les écoles secondaires genevoises, pour autant que ces pratiques s'inscrivent dans une vision actualisée de la géographie scolaire et qu'elles tendent vers la réalisation des finalités de la loi sur l'instruction publique.

- C'est enfin un lieu de réflexion, de confrontation d'idées, d'apports sur les nouvelles approches de l'enseignement de la géographie.

Centré sur les candidats, partant toujours de leur expérience et de leurs représentations tant du métier d'enseignant que de la géographie et de son enseignement, le séminaire de méthodologie poursuit une démarche spiralaire.

Dans un premier temps, chaque maître en formation élabore une séquence didactique qui sera réellement utilisée en classe, puis la présente au groupe. La séquence est alors disséquée, discutée et analysée sur plusieurs registres, pour identifier les composantes de tout enseignement. Après ce premier tour d'horizon, une réflexion sur les définition, place et rôle de l'enseignement de la géographie s'impose en principe "naturellement». Cette période, qui s'étend de septembre à novembre, est particulièrement importante: elle permet au formateur conjointement aux visites en classe et aux entretiens individuels - de cerner progressivement les besoins de chaque candidat et de moduler en conséquence tant le suivi pratique de chacun que les contenus du séminaire.

Dans un deuxième temps, les problèmes professionnels soulevés par l'analyse des séquences didactiques sont repris un à un, selon des priorités définies avec les candidats. Conçu dans une optique interactive et appropriative, le séminaire n'est pas destiné à fournir des «recettes». Il doit permettre à l'enseignant, à propos de chaque thématique, de confronter ses représentations à celles des autres maitres de son groupe de formation, de rapporter ses expériences, d'intégrer de nouvelles connaissances, de se poser des questions essentielles susceptibles tant d'aiguiser sa réflexion didactique que de favoriser une diversification de ses pratiques à court, moyen ou long terme. Les contenus traités - leur ordre et leur importance dépendant des priorités évoquées - s'inscrivent dans des registres variés tels que l'observation d'une leçon, l'auto-évaluation de l'enseignant, les objectifs de la géographie à l'école, les styles et méthodes d'enseignement et d'apprentissage, l'intégration des moyens audiovisuels, l'utilisation de la presse ou des jeux de simulation, l'éducation au développement, les divers types d'évaluation, les modes de communication, l'enseignement dans les classes non prégymnasiales, les travaux de groupe, l'utilisation des représentations, l'apprentissage assisté par ordinateur.

Tout au long de la première année, les apports du séminaire de méthodologie s'articulent aussi étroitement que possible à ceux de didactique générale et de pédagogie, et dans une moindre mesure, à ceux de psychologie et de sociologie. Actuellement, les candidats géographes sont regroupés, pour ces séminaires, avec les candidats historiens. Ainsi, en didactique générale et pédagogie, ils sont amenés à continuer, dans une perspective transdisciplinaire, le travail de théorisation de leur pratique entamé en méthodologie.

Autre facette de la méthodologie de la géographie: les visites du (des) méthodologue(s) en classe. Annoncées ou non - selon les désirs de chaque candidat - mais régulières, elles permettent autant d'observer les capacités du candidat à ètablir une relation pédagogique harmonieuse ou à gérer des situations relationnelles conflictuelles que de voir comment le maître met en place des situations d'apprentissage différenciées et adaptées. Voulues par essence formatives, elles sont toujours suivies d'un entretien avec le candidat visité. Cet entretien est primordial, puisqu'il permet de confronter la vision de la leçon du "visité» et du "visiteur»; de cette interaction critique et en fonction des besoins «réels» (du moins, tels que perçus par le formateur) peut naitre un programme de formation plus individualisé, adapté au contexte et au niveau de compétences de chacun. Le travail du formateur consiste également à articuler ses observations dans les classes des candidats aux réflexions menées durant le séminaire de méthodologie.

\section{Quelques remarques}

- De nombreux suppléants en géographie ont dû attendre plusieurs années avant de pouvoir entamer leur formation pédagogique. Aussi, les candidats géographes qui suivent actuellement une formation aux ètudes pédagogiques comptent tous cinq ans ou plus d'expérience de l'enseignement. C'est dire que la formation dispensée relève moins du b.a.BA nécessaire à l'enseignant débutant que de la formation continue. Toutefois, pour certains maîtres, ces deux années correspondent à une remise en question fondamentale de leur enseignement, voire de leur propre image d'enseignant.

- Actuellement, le taux de couverture des heures de géographie par des maîtres nommés ou garantis d'emploi approche le $100 \%$. A Genève, la géographie est presque entièrement enseignée par des "professionnels» de la discipline. Compte tenu du faible nombre de départs, il n'y aura plus beaucoup de maitres en formation initiale ces prochaines années. 
- Dès l'année scolaire 1994-1995, tout nouveau suppléant est dorénavant astreint à une formation élémentaire d'une année, condition liminaire à une poursuite de la formation ultérieurement aux études pédagogiques.

- Tant par ses structures de "groupes de maîtres» d'une même branche par établissement ou par type d'école que par son offre particulièrement étoffée en matière de formation continue, Genève permet à ses enseignants d'envisager la réflexion pédagogique de manière «continuée». En géographie, depuis la rentrée de septembre 1994, une formation spécifique intitulée "Pratiques pédagogiques en géographie» réunit régulièrement trente-six enseignants du cycle d'orientation. Alternant apports théoriques de personnes-ressources, moments de réflexion théorico-pratique par groupe de douze guidée par un animateur-coordinateur et phases d'expérimentation en classe de nouvelles approches, ce modèle de formation semble correspondre aux attentes et besoins de nombreux enseignants désireux de se "professionnaliser» toujours davantage. 